\title{
Primer registro de Najas marina (Hydrocharitaceae) y listado florístico de hidrófitas sumergidas en el Bajo Paraná (Argentina)
}

First record of Najas marina (Hydrocharitaceae) and floristic check list of the submersed hydrophytes in the Lower Paraná River (Argentina)

\author{
Brumnich, Federico ${ }^{1 *}$; Berenice Schneider ${ }^{2}$ \\ 1 Instituto Argentino de Nivología, Glaciología y Ciencias Ambientales (IANIGLA), Consejo \\ Nacional de Investigaciones Científicas y Técnicas - Universidad Nacional de Cuyo - Go- \\ bierno de Mendoza (CONICET-UNCuyo-Gob.Mza.). Av. Ruíz Leal s/n, Parque General San \\ Martín, (5500) Mendoza, Argentina. \\ 2 Instituto Nacional de Limnología (INALI), Consejo Nacional de Investigaciones Científicas \\ y Técnicas - Universidad Nacional del Litoral (CONICET-UNL). Ciudad Universitaria, Paraje \\ El Pozo s/n, (3000) Santa Fe capital, Santa Fe, Argentina. \\ * Autor corresponsal: federicobn@gmail.com
}

\section{RESUMEN}

Se comunica el registro de Najas marina L., una hidrófita completamente sumergida, en el Bajo Paraná (Argentina) como nueva cita para su flora y se revisan brevemente aspectos de su ecología (rasgos biológicos, hábitat y distribución). Además, se presenta un listado de las 24 especies de hidrófitas sumergidas (en 12 familias y 15 géneros) que han sido registradas para la extensa planicie de inundación del Bajo Paraná.

Palabras clave - Delta del Paraná; macrófitas acuáticas; nueva cita; Paraná Medio; planicie de inundación.

Ref. bibliográfica: Brumnich, F.; Schneider, B. 2020. "Primer registro de Najas marina (Hydrocharitaceae) y listado florístico de hidrófitas sumergidas en el Bajo Paraná (Argentina)". Lilloa 57 (1): 13-35. Fundación Miguel Lillo, Tucumán, Argentina. D.O.I.: doi.org/10.30550/j.lil/2020.57.1/2

- Recibido: 10/02/20 - Aceptado: 05/05/20

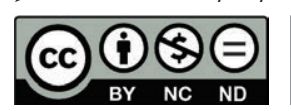




\begin{abstract}
Najas marina L., a completely submersed hydrophyte, is communicated as a new record for the flora of the Lower Paraná River floodplain (Argentina), and aspects relative to its ecology (biological traits, habitat and distribution) are briefly reviewed. Additionally, a check list of the 24 submersed hydrophyte species (12 families, 15 genera) recorded in the large Lower Paraná floodplain is provided.
\end{abstract}

Keywords - Aquatic macrophytes; floodplain; Middle Paraná River; new record; Paraná Delta.

\title{
INTRODUCCIÓN
}

El género Najas L. (Hydrocharitaceae) incluye 30-40 especies de hidrófitas de distribución subcosmopolita en sistemas acuáticos continentales, con centros de diversidad en los trópicos de Asia y África (Haynes, Holm-Nielsen y Les, 1998; Haigh, 2009; Ito, Tanaka, Gale, Yano y Li, 2017). Para la región Neotropical, la de mayor riqueza de hidrófitas en el planeta, se citan seis especies nativas y una naturalizada (Lowden, 1986; Chambers, Lacoul, Murphy y Thomaz, 2008; Haigh, 2009), mientras que para la Flora del Conosur de Sudamérica se registran tres especies nativas (Zuloaga, Belgrano y Zanotti, 2019), todas presentes en humedales de la alta cuenca del río Paraná pero, hasta este trabajo, sin registros en humedales del Bajo Paraná (Neiff, 1986; Pott y Pott, 2000; Ferreira, Mormul, Thomaz, Pott y Pott, 2011; Zuloaga et al., 2019).

Los paisajes de humedales del Bajo Paraná se caracterizan por una gran diversidad de plantas adaptadas a tolerar los pulsos de inundación y estiajes, sobresaliendo las formas de vida hidrófita, que presentan rasgos contrastantes, e.g., con hojas en distinta posición respecto a la columna de agua: sumergidas, flotantes o emergentes (Neiff y Malvárez, 2004; Minotti, Ramonell y Kandus, 2013). Dicha diversidad se refleja en el hecho de que solo en ca. $10 \%$ de la planicie de inundación del Bajo Paraná, correspondiente a los territorios de las provincias de Santa Fe y Entre Ríos, se han registrado aproximadamente un quinto de las especies de plantas vasculares de cada una de tales provincias (Aceñolaza et al., 2008a). Aun así, son esperables nuevos registros florísticos teniendo en cuenta, principalmente, el carácter de corredor biogeográfico del río Paraná (Oakley, Prado y Adámoli, 2005) y la menor continuidad en el estudio de su flora comparado con ambientes terrestres adyacentes.

En el marco de un proyecto de investigación sobre metacomunidades en la planicie del Bajo Paraná se coleccionó material de $N$. marina en una laguna aislada de cauces activos. El objetivo de este trabajo es comunicar a $N$. marina como nueva cita para la flora del Bajo Paraná junto a una reseña ecológica de la especie; y, adicionalmente, aportar un listado de todas las especies de hidrófitas sumergidas registradas para el área. 


\section{MATERIALES Y MÉTODOS}

El río Paraná es uno de los 10 mega ríos del planeta, dado su caudal medio anual mayor a $17000 \mathrm{~m}^{3} / \mathrm{s}$ (Latrubesse, 2008). La planicie de inundación del Bajo Paraná ocupa $c a .35000 \mathrm{~km}^{2}$ de humedales que se extienden entre la confluencia de los ríos Paraná y Paraguay y la desembocadura del Paraná en el río de la Plata (Neiff, 1986; Ramonell, Kandus, Minotti, Borro y Morandeira, 2012; Alberdi, Ramonell y Pereira, 2018). Vale aclarar que el Bajo Paraná incluye dos sectores: el Paraná Medio (Alberdi et al., 2018) y el "Delta del Paraná" sensu lato, i.e., el complejo fluvio-litoral del río Paraná en su desembocadura (Ramonell et al. 2012). El Bajo Paraná se caracteriza por una alta heterogeneidad tanto espacial, en términos geomorfológicos, como temporal, con pulsos de inundación y estiaje, donde los niveles hidrométricos oscilan de 3 a $4 \mathrm{~m}$, con un caudal histórico que ha variado desde 8000 a $60000 \mathrm{~m}^{3} / \mathrm{s}$, aproximadamente (Aceñolaza, Zamboni, Sione y Kalesnik, 2008b; Alberdi et al., 2018; Marchetti, Ramonell, Brumnich, Alberdi y Kandus, 2020).

El material estudiado de N. marina proviene de una laguna del Bajo Paraná, situada en cercanías de las ciudades de Santa Fe y Paraná (Fig. 1A). Al momento del muestreo, la zona litoral de la laguna presentaba una gran cobertura de macrófitas, con dominancia de flotantes (Fig. 1B) mientras que en la zona limnética se registraron macrófitas sumergidas y algas verdes filamentosas (Fig. 1 C y D). El muestreo de esta laguna formó parte de una serie más amplia de muestreos, de un total de 19 lagunas y otros 12 cuerpos de agua con diferente conectividad a cauces activos. Los muestreos fueron cuatro (dos en inundación y dos en estiaje), entre noviembre de 2013 y marzo de 2016 (para más detalles geográficos e hidro-geomorfológicos sobre el área de estudio ver: Mayora, Scarabotti, Schneider, Alvarenga y Marchese, 2020). En campo se midieron variables físico-químicas: conductividad y $\mathrm{pH}$ (con medidor portátil de agua Hanna@); profundidad (con varas graduadas y linterna batimétrica); y transparencia (con disco de Secchi). La cobertura de hidrófitas se registró a través de cuadrados de $1 \mathrm{~m}$ de lado, colocados a lo largo de transectas ubicadas perpendicularmente a la línea de costa. Se realizaron colectas de material botánico que fueron observadas con lupa (estéreo-microscopio), tanto en fresco, como posterior a su herborizado; los ejemplares fueron depositadas en los herbarios DTE y STL (Thiers, 2020).

Para la determinación botánica de $N$. marina se siguió principalmente la revisión de Lowden (1986) entre otras obras (Triest, 1987, 1988; Talavera y Gallego 2010; Wang, Youhao, Haynes y Hellquist, 2010) y se comparó el material estudiado con imágenes de exsiccata de CTES, LP, K, MO, OS, P (Thiers, 2020). Para la descripción se consideró lo reportado por diferentes autores (Lowden, 1986; Triest, 1987, 1988; Urquiola Cruz, Vega Hernández, Novo Carbó y López, 1998; Talavera y Gallego, 2010; Wang et al. 2010) en relación a observaciones propias sobre el material del presente estudio. Para confirmar su distribución en el Bajo Paraná se consultaron herbarios con colecciones en el área: CTES, CTESN, DTE, ERA, MFA, MNES, PAR, SF, UNR (Thiers, 2020). Para la reseña ecológica se analizaron publicaciones sobre la biología y el hábitat de $N$. marina que se compararon con registros sobre la población y el ambiente del material estudiado. 


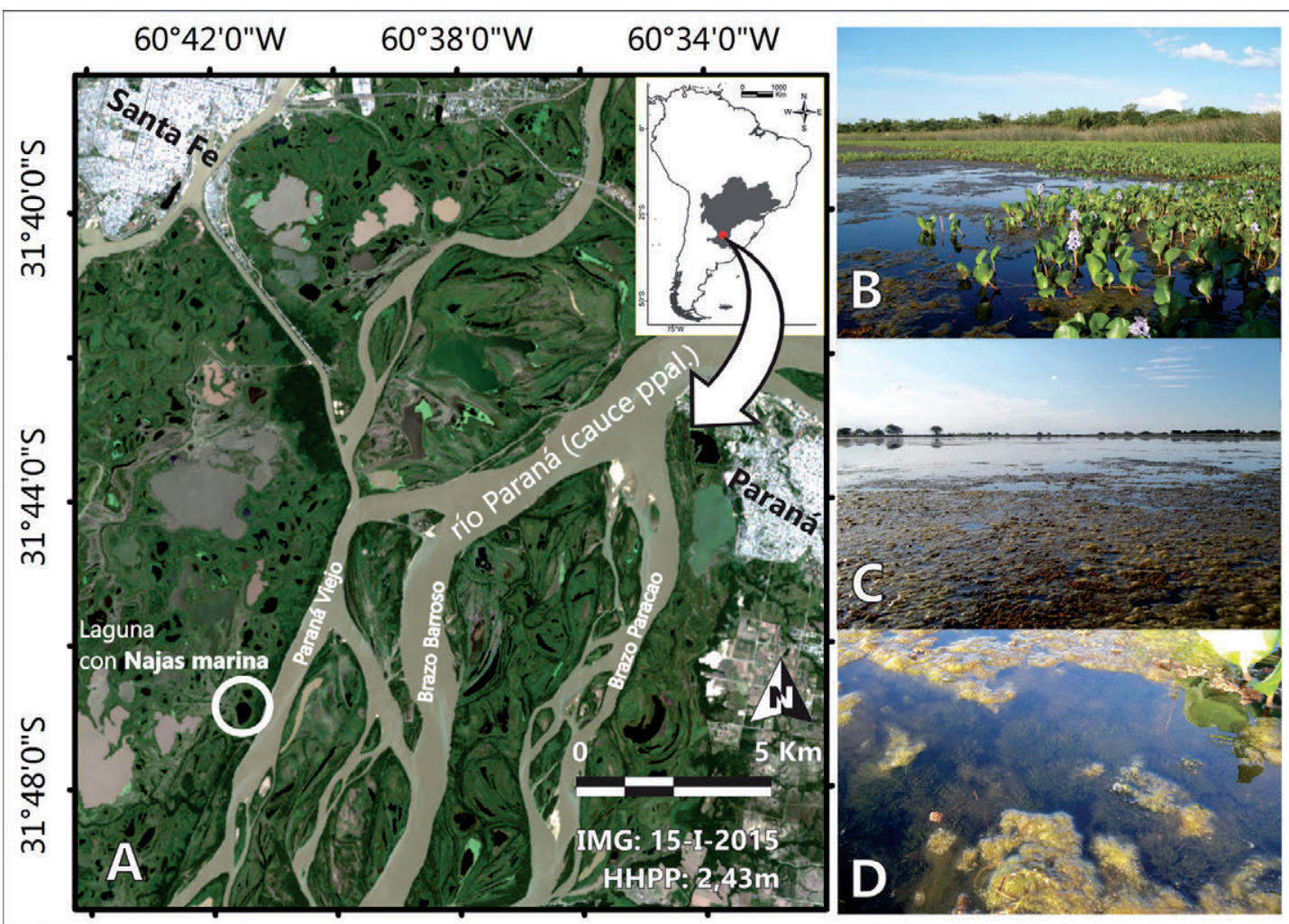

Fig. 1. A) Laguna aislada (círculo), hábitat del material de estudio, en cercanía de las ciudades de Santa Fe y Paraná, en el contexto de la cuenca del río Paraná (IMG = fecha de imagen Landsat 8 OLI; HHPP = altura hidrométrica en el Puerto de la ciudad de Paraná, indicando una fase de aguas bajas). B) Zona litoral de la laguna. C) Zona limnética de la laguna. D) Detalle de la población de $N$. marina estudiada, sumergida, cubierta de algas Zygnemataceae.

Fig. 1. A) Isolated shallow lake (circle), habitat of the study material, near the cities of Santa Fe and Paraná, in the context of the Paraná River basin (IMG=Landsat 8 OLI image date; HHPP= hydrometric level in the Paraná city gauge station, indicating a low water phase). B) Littoral zone of the shallow lake. C) Limnetic zone of the shallow lake. D) Details of the studied N. marina population, submersed, covered with Zygnemataceae algae.

Para la revisión bibliográfica sobre la ocurrencia de las hidrófitas sumergidas presentes en el Bajo Paraná se tuvieron en cuenta dos aspectos. Por un lado, se consideró como hidrófitas sumergidas a las especies macróscópicas, herbáceas y con la mayor parte de su biomasa sumergida, ya que son las únicas especies que, en estado maduro, habitan frecuentemente la columna de agua (i.e., no solamente durante la fase de inundación). Por otro lado, se analizaron tres tipos de fuentes de información: (i) revisiones florísticas de distintos sectores del área (e.g., Neiff, 1986; Madanes, Quintana, Kandus y Bó, 2015); (ii) listas florísticas originales, i.e., tesis, publicaciones no asociadas a tesis (para evitar pseudo-réplicas de tesis) e informes inéditos, desde Morello (1949) al presente; (iii) diferentes tratamientos taxonómicos (monografías/revisiones, capítulos de Floras regionales, etc.). Todas las fuentes se citan en el Apéndice 1. 


\section{RESULTADOS}

Najas marina L., Species Plantarum: 1015. 1753.

Lectotipo (LINN 1156.1) designado por Viinikka Y. (1976).

Annales Botanici Fennici 13: 128.

Fig. 2: A-G

Hierba acuática, totalmente sumergida, arraigada, anual. Tallos de $30-100 \mathrm{~cm}$ long., quebradizos, algo ramificados en la parte superior, diámetro de 0,3-2 (-4,5) mm, entrenudos densamente espinosos hasta inermes, los inferiores con raíces adventicias no ramificadas asemejándose a un rizoma. Hojas subopuestas, en apariencia verticiladas en los nudos superiores, sésiles, linear-lanceoladas a filiformes, con dientes marginales, (8-) 12-30 (-35) mm long. $\times$ 1,3-3 (-4) mm lat. -incluidos los dientes-, vainas algo redondeadas enteras o con dientes inconspícuos, ápice con 1 (2) dientes espinulosos, en general con dientes en el nervio medio del hipófilo; dientes trian-

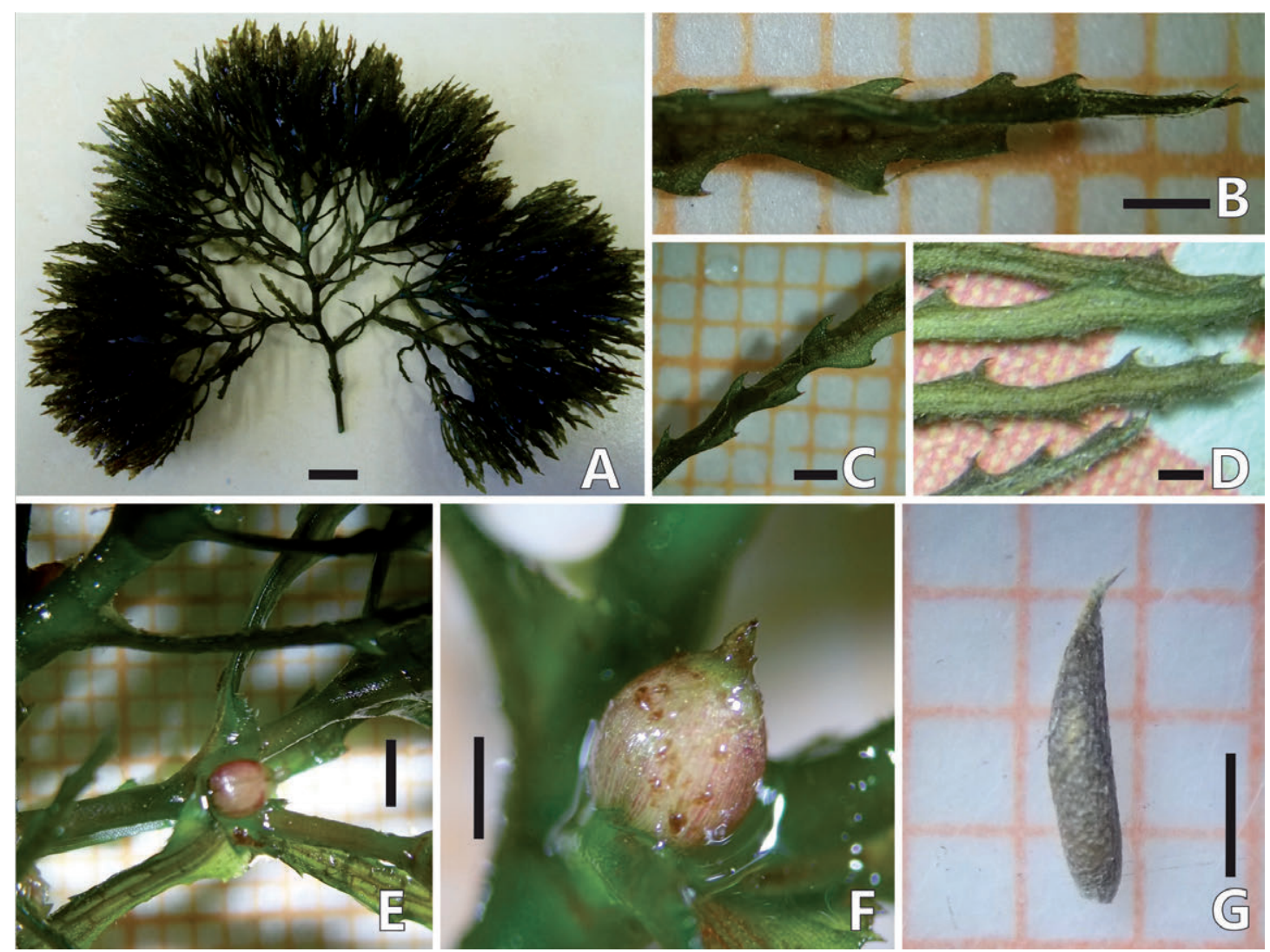

Fig. 2. Exomorfología del material estudiado de Najas marina en la planicie de inundación del Bajo Paraná. A) Aspecto de la ramificación apical. B-D) Detalles de hojas con dientes en las márgenes y en el hipófilo. E-G) Detalles del fruto axilar con pericarpo persistente. Las barras de escala corresponden a $1 \mathrm{~cm}(A), 1 \mathrm{~mm}$ (B, C, G) y $2 \mathrm{~mm}$ (E, F). Schneider 40, 42 (STL), 41 (DTE).

Fig. 2. Exomorphology of the Najas marina materials from the Lower Paraná floodplain. A) Appearence of the apical branch. B-D) Details of leaves with teeth in the margins and in the hypophyll. E-G) Details of the axillary fruit with persistent pericarp. Scale bars correspond to $1 \mathrm{~cm}(A), 1 \mathrm{~mm}$ (B, C, G) and $2 \mathrm{~mm}$ (E, F). Schneider 40, 42 (STL), 41 (DTE). 
gulares, foliáceos, terminados en una espínula pardusca o rojiza. Plantas dioicas con flores axilares, inconspícuas. Flores estaminadas solitarias, encerradas en una bráctea espatiforme, pediceladas en la antesis, periantadas, 1-estaminadas, anteras subsésiles, elipsoidales, 4-tecadas, de dehiscencia apical. Flores pistiladas solitarias o geminadas, sésiles, perianto persistente, $c a$. 2-4 mm long., estigma (2-) 3 (-4) -fido. Fruto aquenio, con pericarpo fino, membranoso, persistente. Semillas (3,2) 3,5-5 mm long. $\times$ $(1,3)$ 1,5-2,5 (3) mm lat., \pm ovoides o elipsoidales, pardas, con fovéolas poligonales \pm cuadrangulares dispuestas en filas longitudinales irregulares.

\section{Material estudiado}

ARGENTINA. Prov. Santa Fe, Dpto. La Capital, 31046'56,7'S 6041'25,98'O (laguna "Los Gansos", aislada de cauces activos, a unos $300 \mathrm{~m}$ del brazo Paraná Viejo), ca. $5 \mathrm{~m}$ snm, 29-XI-2013 (fr), Schneider 40 (STL). Ibidem, 03-IV-2014 (fr), Schneider 41 (DTE), Schneider 42 (STL). (Figs. 1 y 2).

\section{Iconografía}

Hunziker (1966: p. 223), ilustración de H. García (disponible en Flora del Conosur, Zuloaga et al., 2019); Lowden (1986: p. 160); Urquiola Cruz et al. (1998: p. 87); Arbo, López, Schinini y Pieszko (2002: p. 21), ilustración de L. Gómez; Zhang (2010: p. 100), ilustración de C. Baolian.

\section{DISCUSIÓN}

\section{Observaciones morfológicas y taxonómicas adicionales}

$\mathrm{Al}$ igual que otras hidrófitas, $N$. marina es reconocida como una especie muy polimorfa (Triest, 1988; Talavera y Gallego, 2010). Los ejemplares estudiados no presentan dientes en los entrenudos (Fig. 2A), algo que generalmente no se ha representado en la iconografía (la excepción está en Arbo et al., 2002: p. 21). Este rasgo de valor diagnóstico (Triest, 1988) no ha sido mencionado por Lowden (1986) para la región Neotropical, pero sí se menciona en la Flora de China (Wang et al., 2010) y la Flora Ibérica (Talavera y Gallego, 2010).

La sinonimia de N. marina y problemas de su tipificación, así como la grafía del propio género Najas (llamado Naias en ocasiones), es tratada en Lowden (1986), Talavera y Gallego (2010) y Bräuchler (2015). Asimismo, Govaerts (2020) compiló ocho nombres infra-específicos aceptados para $N$. marina (cuatro de subespecies y cuatro de variedades). De estos nombres, $N$. marina L. subsp. marina corresponde al único taxón que se distribuye en el hemisferio occidental y se ha descripto con tallo liso (Talavera y Gallego, 2010), un rasgo que se corresponde con el material estudiado 
para el presente trabajo y con parte de los ejemplares coleccionados en Argentina, a saber: Arbo 7950 y 8033 (CTES), Neiff 320 y 1191 (CTES), Ruiz Leal 8344 (MERL), Schinini 11978 (MBM).

\section{Rasgos biológicos y hábitat}

Naja marina se ha registrado en ambientes acuáticos continentales naturales como arroyos, esteros y lagunas (e.g. Hunziker, 1966; Nicora, 1968; Tur, 1970; Lowden, 1986; Neiff, 1986; Arbo et al., 2002). Como contraste vale destacar que el primer registro en la Flora Argentina (y del Conosur), que data de 1944 (Ruiz Leal 8344), es en el lago del Parque San Martín (Mendoza), i.e. un ambiente altamente antropizado.

Las plantas de N. marina pueden desarrollarse en sustratos con una amplia escala granulométrica (desde arenas hasta arcillas) e incluso sobre cúmulos de materia orgánica; sin embargo, la cohesividad del sedimento sería un factor clave en su enraizamiento y por tanto en la colonización efectiva del ambiente (Triest, 1988; Handley y Davy, 2002). Respecto a las condiciones fisicoquímicas del agua, varios autores mencionan a esta especie como tolerante a una alta salinidad (óptimo de hasta $55 \mathrm{mM}$ de $\mathrm{NaCl})$, alta conductividad (195-1250 $\mu \mathrm{S} / \mathrm{cm})$, y un $\mathrm{pH}$ básico del agua (e.g., Agami, Eshel y Waisel, 1984; Triest, 1988; Stanković et al., 2009). Lo reportado se corresponde parcialmente con lo registrado en la laguna "Los Gansos", donde medimos valores puntuales de conductividad $=117.8 \mu \mathrm{S} / \mathrm{cm} ; \mathrm{pH}=9.64$; profundidad $=92 \mathrm{~cm} ; \mathrm{y}$ transparencia $>30 \mathrm{~cm}$.

Para humedales del Alto Paraná, $N$. marina fue mencionada entre las hidrófitas raras que, a su vez, pueden ser abundantes localmente, dependiendo de las condiciones de transparencia, profundidad y sustrato (Neiff 1986). Esto concuerda con nuestras observaciones en el Bajo Paraná donde la especie tuvo una rareza a escala de paisaje, por ser registrada en solo una de 19 lagunas estudiadas en detalle (Mayora et al., 2020); y una dominancia a escala local, por presentar alta cobertura en la zona limnética de tal laguna aislada de un cauce activo (Fig. 1 C-D). Para el Alto Paraná $N$. marina ha sido citada como especie indicadora de la fase de aguas bajas (Neiff, Casco, Mari, Di Rienzo y Poi, 2014), lo que es similar a la altura del río durante nuestra colección del material estudiado (nivel de aguas medias-bajas, $c a .3 \mathrm{~m}$ en el puerto de la ciudad de Paraná).

Sobre las plantas de $N$. marina muestreadas se observó una cubierta densa de algas verdes filamentosas (de la familia Zygnemataceae). Esta asociación también fue señalada para Najas affinis Rendle (sub. N. microcarpa K. Schum.) en el Pantanal (Pott y Pott, 2000).

Naja marina es la única especie dioica del género y las plantas con flores estaminadas son consideradas raras en climas templados (Handley y Davy, 2000). En tal sentido, los ejemplares colectados y estudiados fueron únicamente de plantas femeninas, con frutos. La polinización es hipo-hidrófila y ha sido descripta en detalle, e incluye períodos relativamente prolongados de dispersión de polen, el cual, además, presenta una alta viabilidad en el medio acuoso (Huang, Guo, Robert, Shi y Sun, 2001). La dispersión es hidrócora y probablemente exozoócora; se discute si 
su semilla resiste la endozoocoria, la cual se reportó para patos (Anatidae) y peces actinopterigios (Cirujano y López Alberca, 1984; Agami y Waisel 1986, 1988; Haynes et al., 1998).

\section{Distribución}

El rango biogeográfico de $N$. marina se considera subcosmopolita, ya que ocurre en siete de las ocho regiones del planeta con plantas acuáticas vasculares; i.e., ausente en la Antártida (Chambers et al., 2008; Ito et al., 2017). En la región Neotropical fue citada para 17 países (Lowden, 1986). Dentro del Conosur solo es citada para Argentina (i.e., sin registros en Uruguay, Paraguay, Chile y los tres estados más australes de Brasil), en las provincias políticas de Mendoza, San Luis, Corrientes (Zuloaga et al., 2019), y desde esta contribución, Santa Fe y Misiones (Fig. 3). Vale destacar que Misiones, (provincia no reportada en la Flora del Conosur), fue mencionada por Tur (1970) sin citar los ejemplares Tur (LP 4924) y Tur (MFA 7325), los cuales fueron observados para el presente trabajo. Dado que el Bajo Paraná se sitúa en el territorio de cinco provincias argentinas (Chaco, Corrientes, Santa Fe, Entre Ríos y Buenos Aires), sería esperable hallar a $N$. marina en las provincias donde aún no ha sido citada. Asimismo, vale destacar que la distribución de $N$. marina en el Conosur se corresponde con cuatro provincias biogeográficas (Fig. 3): Monte, Pampean, Chaco y Paraná Forest (sensu Arana, Martinez, Oggero, Natale y Morrone, 2017).

En el Alto Paraná se han registrado tres especies de Najas: N. affinis Rendle (sub. N. microcarpa K. Schum.); N. conferta (A. Braun) A. Braun (Ferreira et al., 2011); y N. marina (Neiff, 1986; Fig. 3). Para el Bajo Paraná, previo al presente registro de $N$. marina, se reportó a $N$. minor All., a partir de un ejemplar coleccionado en la "laguna Guadalupe" (= laguna Setúbal, ciudad de Santa Fe) durante la "gran creciente de 1965/1966" (Tur, 1970). Esta especie, si bien fue citada por Grisebach para Argentina (1879: 282), fue excluida por Múlgura (1996) de la Flora Argentina y, en consecuencia, de la Flora del Conosur. Además, no fue citada para la región Neotropical (Lowden, 1986) y su distribución se restringe al hemisferio norte (Talavera y Gallego, 2010; Govaerts, 2020). No obstante, se comparó el material atribuido a N. minor (Tur s.n. MFA 7327) con otros pertenecientes a $N$. marina (MFA 7325), también coleccionados por Tur en la provincia de Misiones en 1970, ya que ambos materiales se encuentran conservados en formol por períodos de tiempos similares. A pesar de que no se hallaron frutos en el ejemplar atribuido a $N$. minor, sí se registró una diferencia vegetativa considerada diagnóstica (mencionadas en diferentes claves, e.g. Talavera y Gallego 2010 y Wang et al. 2010): la ausencia de dientes espinulosos sobre la nervadura en el hipófilo en " $N$. minor", las cuales sí pueden distinguirse en el material de $N$. marina. Otra diferencia es que los ejemplares de " $N$. minor" se encontraban decolorados mientras el ejemplar de $N$. marina conserva un color verde muy similar a la planta viva; probablemente debido a que el material de " $N$. minor" corresponde a fragmentos de plantas en degradación al momento de ser coleccionados. Por ambas diferencias se considera que el material atribuido a N. minor (MFA 7327) debiera analizarse en futuros estudios del género Najas para confirmar su identidad botánica. 


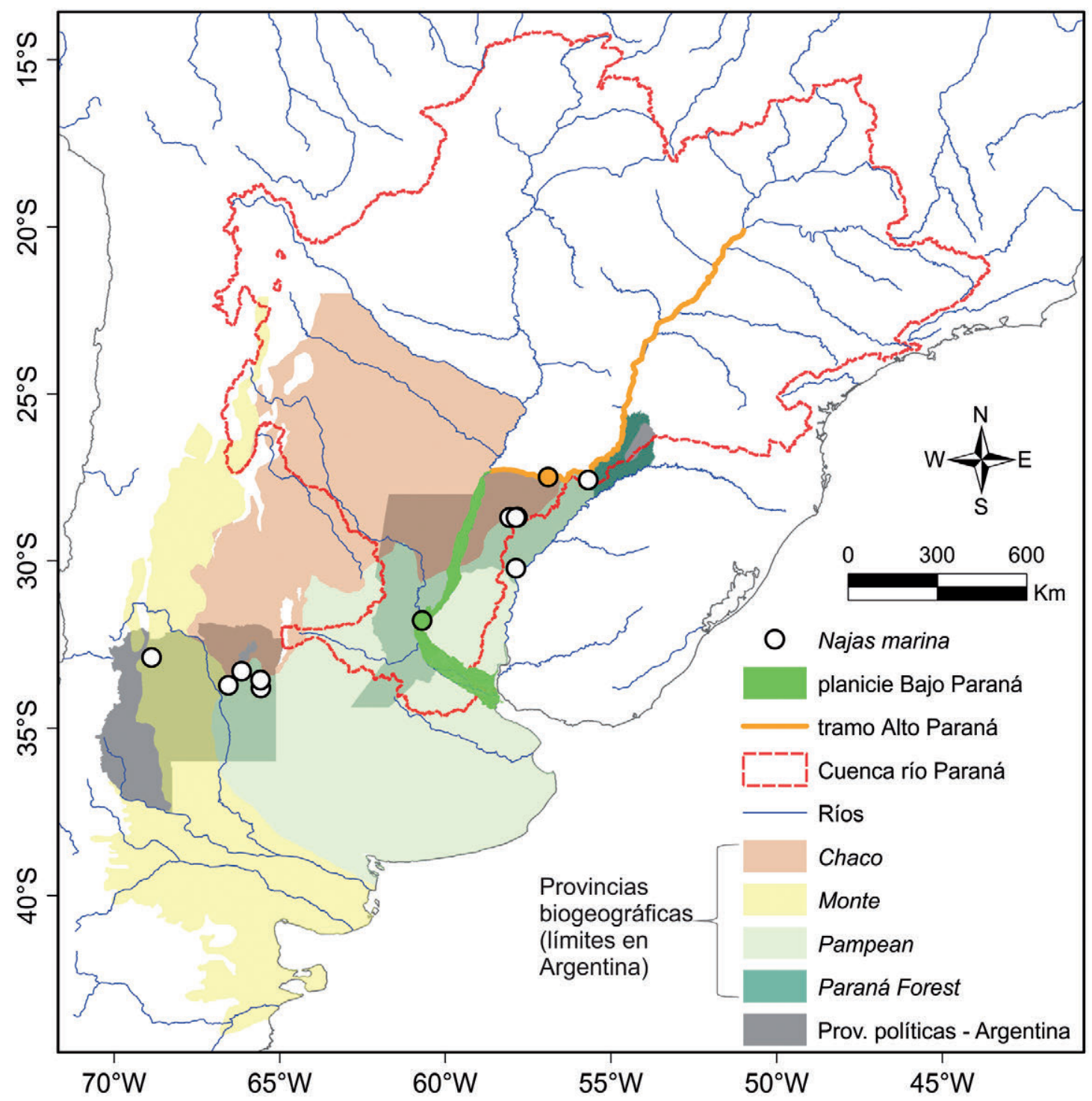

Fig. 3. Registros de Najas marina en Sudamérica Austral; se destacan las ocurrencias para la planicie de inundación del Bajo Paraná (verde) y Alto Paraná (naranja). Además, se señalan las cuatro provincias biogeográficas (sensu Arana et al., 2017) y cinco provincias políticas de Argentina donde se ha registrado la especie (en orden cronológico: Mendoza, San Luis, Corrientes, Misiones y Santa Fe).

Fig. 3. Najas marina records in Southern South America; occurrences for the Lower Paraná floodplain (green) and the Upper Paraná (orange) are highlighted. Also, the four biogeographical provinces (sensu Arana et al., 2017) and five political provinces of Argentina where the species has been registered are pointed out (in chronological order: Mendoza, San Luis, Corrientes, Misiones, and Santa Fe).

Asimismo, es relevante mencionar la posibilidad de hibridación entre las especies de Najas lo que dificulta su reconocimiento por caracteres exomorfológicos (Triest, 1988; Les et al. 2015). Puntualmente, N. marina se ha propuesto recientemente como un complejo de especies (Bräuchler, 2015; Ito et al., 2017), el cual podría comprender dos especies crípticas aisladas reproductivamente por caracteres genómicos y sin diferencias morfológicas claras (López Peredo, Les, Benoit y Tippery, 2011). 


\section{Hidrófitas sumergidas en el Bajo Paraná}

Se han reportado unas 100 especies de hidrófitas herbáceas en la planicie del Bajo Paraná (considerando solamente plantas vasculares). Esta cifra se basa en la revisión de Neiff (1986) con adiciones en base a listas florísticas originales y otras fuentes (citadas en el Apéndice 1). Del total mencionado, hallamos que el número de hidrófitas sumergidas asciende a 24 especies (en 12 familias y 15 géneros), listadas en la Tabla 1. Entre las 24 especies, 17 son citadas como sumergidas de modo permanente y otras 7 han sido reportadas como "semisumergidas", i.e., sumergidas facultativas, tolerantes a desarrollarse con sus hojas en diferente posición respecto a la columna de agua. La persistencia de tal composición de 24 especies es dispar ya que, por un lado, en 15 especies, las colecciones más recientes se realizaron hace 5 o más décadas, por lo que su presencia actual en el Bajo Paraná sería dudosa; y, por otro lado, en las 9 especies restantes, las últimas colecciones ocurrieron en las últimas 2 décadas (Tabla 1 ).

La especie más semejante a $N$. marina, en cuanto a la disposición/tamaño de sus estructuras reproductivas y al aspecto general (observado en campo), es Ceratophyllum demersum, pero esta presenta hojas dicotómicamente divididas y no está arraigada al sustrato, mientras que $N$. marina posee hojas enteras, dientes espinulosos en el nervio medio del hipófilo y permanece arraigada, aunque posee tallos muy quebradizos. Este rasgo vegetativo del hipófilo también sirve para distinguir a $N$. marina del resto de las especies listadas en la Tabla 1.

Por último, consideramos que serán necesarios nuevos muestreos en toda la extensión del Bajo Paraná a fin de lograr un mayor grado de precisión en la evaluación sobre la composición y la persistencia de las poblaciones de hidrófitas de esta gran planicie aluvial.

\section{AGRADECIMIENTOS}

A Nuncia M. Tur, María E. Múlgura, Sylvina L. Casco y Manuel J. Belgrano por sus comentarios al inicio de este trabajo. A Mercedes Marchese por la evaluación crítica del manuscrito en un primer borrador y Melina Devercelli por la determinación botánica de la familia del alga. A la/os trabajadores de los herbarios consultados: G. Pieszko (CTES), D. Iriart (CTESN), D. Reynoso (ERA), L. Iharlegui (LP), A. Bosisio y G. Martínez Schreier (MFA), L. Bonjour y R. Kiesling (MERL), G. Ibargoyen y M.L. Calderón (PAR), E. Exner (SF), L. Galetti y D. Prado (UNR). A la/os evaluadores anónimos por sus comentarios enriquecedores. Este estudio se enmarcó en el proyecto PICT 2012-2095 financiado por la Agencia Nacional de Promoción Científica y Tecnológica. El Consejo Nacional de Investigaciones Científicas y Técnicas financia las becas de los autores de este trabajo. 
Tabla 1. Lista de taxones de hidrófitas sumergidas registradas para la planicie de inundación del Bajo Paraná. En la columna "Especie" se lista el nombre botánico aceptado y, en ciertos casos, el sinónimo (nombre botánico frecuentemente usado en la bibliografía del Apéndice 1) se indica entre corchetes. En la columna "Hábito" se clasifican las especies como sumergidas permanentemente (S) o facultativas (SF). En la columna "Exsiccata" se listan alguno ejemplares botánicos registrados para el Bajo Paraná indicando el herbario donde están depositados (Thiers, 2020). En la columna "Colección" solo se cita la fecha de la última colección en el Bajo Paraná. En negritas se remarcan las 9 especies que fueron colectadas recientemente (ca. últimas 2 décadas); las 15 especies restantes serían citas dudosas ya que su registro más reciente data de ca. 50 o más años atrás.

Table 1. List of registered submerged hydrophyte taxa for the Lower Paraná floodplain. The "Species" column lists the accepted botanical name and, in certain cases, the synonym (botanical name frequently used in the Appendix 1 bibliography) is indicated between square brackets. The "Habit" column classifies the species as either permanently submerged (S) or optional (SF). The column "Exsiccata" lists some botanical specimens registered for the Lower Paraná indicating the herbarium where they are deposited (Thiers, 2020). In the column "Collection" only the date of the last collection in the Lower Paraná is cited. The 9 species that were collected recently (ca. last 2 decades) are highlighted in bold; the remaining 15 species might be doubtful records since their most recent observation dates back to ca. 50 or more years ago.

\begin{tabular}{|c|c|c|c|c|}
\hline Familia & $\begin{array}{c}\text { Especie } \\
\text { [sinónimo frec.] }\end{array}$ & Hábito & Exsiccata & Colección \\
\hline Cabombaceae & $\begin{array}{c}\text { Cabomba caroliniana } \\
\text { A. Gray [Cabomba } \\
\text { australis Speg.] }\end{array}$ & $S^{*}$ & $\begin{array}{l}\text { Tur, N. M. } 1364 \text { (SI } \\
048914) ; \text { Burkart, A. } \\
8953 \text { (SI); Tedone, F. } \\
5264 \text { (SF); Brumnich, } \\
\text { F. } 116 \text { (STL). }\end{array}$ & 2016-01-16 \\
\hline Plantaginaceae & $\begin{array}{l}\text { Callitriche lechleri } \\
\text { (Hegelm.) Fassett }\end{array}$ & SF & $\begin{array}{c}\text { Burkart, A. } 22414 \\
\text { (SI). }\end{array}$ & 1961-11-19 \\
\hline Plantaginaceae & $\begin{array}{c}\text { Callitriche rimosa } \\
\text { Fassett }\end{array}$ & $S$ & $\begin{array}{c}\text { Burkart, A. } 20027 \\
\text { (SI). }\end{array}$ & $1956-09-22$ \\
\hline Ceratophyllaceae & $\begin{array}{l}\text { Ceratophyllum } \\
\text { demersum L. }\end{array}$ & $S$ & $\begin{array}{c}\text { Brumnich, F. } 115 \\
\text { (STL). }\end{array}$ & $2016-01-16$ \\
\hline Hydrocharitaceae & $\begin{array}{c}\text { Egeria densa Planch. } \\
\text { [Elodea densa } \\
\text { (Planch.) Casp.] }\end{array}$ & $S$ & $\begin{array}{c}\text { Scala, A. C. } 40 \text { (LP); } \\
\text { Burkart, A. } 4521 \text { (SI); } \\
\text { Cabrera, A. L. } 10640 \\
\text { (LP). }\end{array}$ & $1950-05-18$ \\
\hline Hydrocharitaceae & $\begin{array}{c}\text { Egeria najas Planch. } \\
\text { [Elodea najas } \\
\text { (Planch.) Casp.] }\end{array}$ & $S$ & Burkart, A. 8929 (SI). & $1938-02-28$ \\
\hline Elatinaceae & $\begin{array}{c}\text { Elatine lorentziana } \\
\text { Hunz. }\end{array}$ & $S$ & Tedone, F. 5108 (SF). & 1967-11-16 \\
\hline Elatinaceae & $\begin{array}{c}\text { Elatine triandra } \\
\text { Schkuhr var. triandra }\end{array}$ & SF & $\begin{array}{l}\text { Tur, N. M. s.n. (SI } \\
\text { 26436). }\end{array}$ & $\begin{array}{l}\text { sin datos; citado en } \\
\text { Bacigalupo (1970). }\end{array}$ \\
\hline Cyperaceae & $\begin{array}{c}\text { Eleocharis } \\
\text { viridans Kük. ex } \\
\text { Osten [Eleocharis } \\
\text { (Heleocharis) prolifera } \\
\text { auct. non Torr.] }\end{array}$ & $S$ & $\begin{array}{c}\text { Burkart, A. 8523, } \\
\text { 12887, } 29770(\mathrm{SI}) ; \\
\text { Nicora, E. G. } 3414 \\
\text { (SI). }\end{array}$ & $\begin{array}{c}\text { 1973-00-00 (sin } \\
\text { datos de mes y día) }\end{array}$ \\
\hline Hydrocharitaceae & $\begin{array}{l}\text { Elodea callitrichoides } \\
\text { (Rich.) Casp. }\end{array}$ & $S$ & $\begin{array}{c}\text { Ragonese, A. E. } 3610 \\
\text { (SF); Schneider, B. } \\
242 \text { (STL). }\end{array}$ & $2019-10-23$ \\
\hline Isoetaceae & $\begin{array}{c}\text { Isoetes ekmanii U. } \\
\text { Weber }\end{array}$ & SF & $\begin{array}{c}\text { Burkart, A. } 4003 \text { (BA } \\
\text { 8231). }\end{array}$ & $1931-09-25$ \\
\hline Apiaceae & $\begin{array}{l}\text { Lilaeopsis attenuata } \\
\text { (Hook. y Arn.) Fernald }\end{array}$ & SF & $\begin{array}{l}\text { Burkart, A. 4004, } \\
3989 \text { (SI). }\end{array}$ & $1931-11-25$ \\
\hline
\end{tabular}


Tabla 1 (cont.).

\begin{tabular}{|c|c|c|c|c|}
\hline Familia & $\begin{array}{c}\text { Especie } \\
\text { [sinónimo frec.] }\end{array}$ & Hábito & Exsiccata & Colección \\
\hline Apiaceae & $\begin{array}{c}\text { Lilaeopsis brasiliensis } \\
\text { (Glaz.) Affolter } \\
\text { [Lilaeopsis minor } \\
\text { (A.W. Hill) Pérez- } \\
\text { Mor.] }\end{array}$ & SF & $\begin{array}{c}\text { Hunziker, A. T. } 6632 \\
\text { (CORD); Pérez- } \\
\text { Moreau s.n. (BA- } \\
\text { 6536). }\end{array}$ & $1946-01-10$ \\
\hline Apiaceae & $\begin{array}{l}\text { Lilaeopsis carolinensis } \\
\text { J.M. Coult. y Rose }\end{array}$ & SF & $\begin{array}{c}\text { Pensiero J.F. } 5420 \\
\text { (SF); Ragonese, A. E. } \\
\text { y Martinez Crovetto } \\
\text { R. N. } 53 \text { (BAB). }\end{array}$ & $1999-12-28$ \\
\hline Haloragaceae & $\begin{array}{c}\text { Myriophyllum } \\
\text { aquaticum (Vell.) } \\
\text { Verdc. [Myriophyllum } \\
\text { brasiliense Cambess.] }\end{array}$ & $\mathrm{S}^{* *}$ & $\begin{array}{c}\text { Morello, J. } 16939 \\
\text { (SI); Burkart, A. } 5086 \\
\text { (SI); Pensiero, J.F. } \\
\text { 5427, } 5542 \text { (SF). }\end{array}$ & $1998-12-28$ \\
\hline Hydrocharitaceae & Najas marina L. & $S$ & $\begin{array}{c}\text { Schneider, B. } 41 \\
\text { (DTE); Schneider, } B . \\
\text { 40, } 42 \text { (STL). }\end{array}$ & 2014-03-04 \\
\hline Potamogetonaceae & $\begin{array}{c}\text { Potamogeton } \\
\text { ferrugineus Hagstr. }\end{array}$ & $S$ & $\begin{array}{c}\text { Parodi, L. R. } 5406 \\
\text { (BAA). }\end{array}$ & 1923-03-16 \\
\hline Potamogetonaceae & $\begin{array}{l}\text { Potamogeton gayi A. } \\
\text { Benn. }\end{array}$ & $S$ & $\begin{array}{c}\text { Scala, A. C. } 54 \text { (LP); } \\
\text { Parodi, L.R. } 5386 \\
\text { (BAA); Capurro, R. H. } \\
\text { s.n. (BA 54751). }\end{array}$ & 1940-11-02 \\
\hline Potamogetonaceae & $\begin{array}{l}\text { Potamogeton } \\
\text { pusillus L. } \\
\text { [Potamogeton } \\
\text { berteroanus Phil.] }\end{array}$ & $S$ & $\begin{array}{c}\text { Burkart, A. } 9218 \text { (S) } \\
\text { citado en Haynes y } \\
\text { Holm-Nielsen (2003).; } \\
\text { Schneider, B. } 144 \\
\text { (STL). }\end{array}$ & 2013-10-16 \\
\hline Ranunculaceae & $\begin{array}{c}\text { Ranunculus } \\
\text { flagelliformis Sm. }\end{array}$ & SF & $\begin{array}{c}\text { Burkart, A. } 5024 \text { (SI); } \\
\text { Burkart, A. } 29879 \\
\text { (SI). }\end{array}$ & 1973-11-08 \\
\hline Lentibulariaceae & $\begin{array}{c}\text { Utricularia foliosa } \\
\text { L. [Utricularia } \\
\text { oligosperma A. St.- } \\
\text { Hil.] }\end{array}$ & $S$ & $\begin{array}{c}\text { Tedone, F. } 5117 \text { (SF); } \\
\text { Morrone, 0. } 5361 \\
\text { (SI). }\end{array}$ & 2005-07-04 \\
\hline Lentibulariaceae & $\begin{array}{c}\text { Utricularia gibba L. } \\
\text { [Utricularia obtusa } \\
\text { Sw.] }\end{array}$ & $\mathrm{S}$ & $\begin{array}{c}\text { Parodi 9515, L. R. } \\
\text { (BAA); Tedone, F. } \\
5116 \text { (SF); Burkart, } \\
\text { A. } 8942,9217 \text { (SI); } \\
\text { Schneider, B. } 142 \\
\text { (STL). }\end{array}$ & $2013-10-23$ \\
\hline Lentibulariaceae & $\begin{array}{l}\text { Utricularia platensis } \\
\text { Speg. }\end{array}$ & $S^{*}$ & $\begin{array}{c}\text { Burkart, A. } 22751 \\
\text { (SI); Tur, N. M. } 1362 \\
\text { (LP); ambos citados } \\
\text { en Dawson (1973) } \\
\text { sub Utricularia inflata } \\
\text { Walter (especie } \\
\text { excluida). }\end{array}$ & $1970-11-27$ \\
\hline Potamogetonaceae & $\begin{array}{c}\text { Zannichellia palustris } \\
\text { L. }\end{array}$ & $S$ & $\begin{array}{c}\text { Hunziker, J.H. } 2336 \\
\text { (LP). }\end{array}$ & $1947-09-28$ \\
\hline
\end{tabular}

* Hojas vegetativas sumergidas, brácteas florales flotantes.

** La mayoría de las hojas sumergidas, las de los entrenudos superiores son emergentes. 


\section{BIBLIOGRAFIA}

Aceñolaza, P., Manzano, A., Rodríguez, E., Sánchez, L., Ronchi, A. L., Giménez, E., Demonte, D. y Marchetti, Z. (2008a). Biodiversidad de la región del complejo deltaico del Río Paraná. Miscelánea INSUGEO 17 (1): 127-152.

Aceñolaza, P., Zamboni, L. P., Sione, W. y Kalesnik, F. (2008b). Caracterización de la región superior del complejo litoral del río Paraná: grandes unidades de ambiente. Miscelánea INSUGEO 17 (2): 293-308.

Affolter, J. M. (1985). A monograph of the genus Lilaeopsis (Umbelliferae). Systematic Botany Monographs 6: 1-140.

Alberdi R., Ramonell C. G. y Pereira, M. S. (2018). El tramo medio del río sus límites, extensión y variabilidad hidrográfica. El ojo del Cóndor 9: 4-9.

Agami, M., Eshel, A. y Waisel Y. (1984). Najas marina in Israel: It is a halophyte or a glycophyte? Physiologia Plantarum 61 (4): 634-636.

Agami, M. y Waisel, Y. (1986). The role of mallard ducks (Anas platyrhynchos) in distribution and germination of seeds of the submerged hydrophyte Najas marina L. Oecologia 68 (3): 473-475.

Agami, M. y Waisel, Y. (1988). The role of fish in distribution and germination of seeds of the submerged macrophytes Najas marina L. and Ruppia maritima L. Oecologia 76 (1): 83-88.

Arana, M. D., Martínez, G. A., Oggero, A. J., Natale, E. S. y Morrone, J. J. (2017). Map and shapefile of the biogeographic provinces of Argentina. Zootaxa 4341 (3): 420-422.

Arbo, M. M. (2002). Las plantas carnívoras. En: M. M. Arbo, S. G. Tressens (Eds.), Flora del Iberá (pp. 155-165). Corrientes: Editorial de la Universidad Nacional del Nordeste.

Arbo, M. M., López, M. G., Schinini, A. y Pieszko G. (2002). Las plantas hidrófilas. En: M. M. Arbo, S. G. Tressens (Eds.), Flora del Iberá (pp. 9-67). Corrientes: Editorial Universitaria de la Universidad Nacional del Nordeste.

Bacigalupo, N. M. (1970). Observaciones sobre el género Elatine L. en la Argentina. Darwiniana 16 (1/2): 106-115.

Bacigalupo, N. M. (1979a). Callitrichaceae. En: A. Burkart (dir.), Flora Ilustrada de Entre Ríos - Parte V B (pp. 577-582). Buenos Aires: Instituto Nacional de Tecnología Agropecuaria.

Bacigalupo, N. M. (1979b). El género Callitriche en la flora argentina. Darwiniana 22 (1-3): 377-396.

Bacigalupo, N. M. (1987). Ranunculaceae. En: N. Troncoso y N. Bacigalupo (Dirs.). Flora Ilustrada de Entre Ríos - Parte III (pp. 305-321). Buenos Aires: Instituto Nacional de Tecnología Agropecuaria.

Bacigalupo, N. M (2005). Elatinaceae. En: N. M. Bacigalupo (dir.), Flora Ilustrada de Entre Ríos - Parte IV B (pp. 358-361). Buenos Aires: Instituto Nacional de Tecnología Agropecuaria.

Barros, M. (1947). Cyperaceae: Scirpoideae: Hypolitrae - Cypereae - Scirpeae (Heleocharis, Scirpus). En: H. R. Descole (Dir.), Genera et Species Plantarum Argentinarum IV (I) (pp. 1-243). Buenos Aires: Kraft. 
Bayo, V., Lallana, V. H., Lorenzatti, E. y Marta, M. C. (1981). Evaluación cuantitativa de la vegetación acuática en las islas del valle aluvial del río Paraná medio. Parte I. Ecología 6: 67-72.

Botta, S. M. (1979). Lentibulariaceae. En: A. Burkart (Dir.), Flora Ilustrada de Entre Ríos - Parte V (pp. 539-547). Buenos Aires: Instituto Nacional de Tecnología Agropecuaria.

Bräuchler, C. (2015). Towards a better understanding of the Najas marina complex: Notes on the correct application and typification of the names $N$. intermedia, $N$. major, and N. marina. Taxon 64 (5): 1028-1030.

Brumnich, F. (2017). Dinámica sucesional de bosques en islas del río Paraná Medio. (Tesis de Licenciatura), Universidad Nacional del Litoral, Argentina.

Burkart, A. (1957). Ojeada sinóptica sobre la vegetación del Delta del Río Paraná. Darwiniana 11 (3): 457-561.

Cabrera, A. L. (1968). Hydrocharitaceae. En: A. L. Cabrera (Dir.), Flora de la provincia de Buenos Aires - Parte I (pp. 305-314). Buenos Aires: Instituto Nacional de Tecnología Agropecuaria.

Capurro, R. H. (1968). División Pteridophyta. En: A. L. Cabrera (Dir.), Flora de la provincia de Buenos Aires - Parte I (pp. 123-146). Buenos Aires: Instituto Nacional de Tecnología Agropecuaria.

Casco, S. L. (2003). Poblaciones vegetales centrales y su variabilidad espacio-temporal en una sección del Bajo Paraná influenciada por el régimen de pulsos. (Tesis Doctoral), Universidad Nacional del Nordeste, Argentina.

Cavallaro, S. y Tobío, M. I. (2017). Fisonomías de vegetación actual en la Hoja Geológica Gualeguaychú. En: G. Lorenz, M. E. Figueroa, A. N. Giannuzzo, M. E. Ludueña (Eds.), III Congreso Argentino de Ecología de Paisajes (pp. 209-213). Santiago del Estero: Editorial de la Universidad Nacional de Santiago del Estero.

Chambers, P. A., P. Lacoul, K. J. Murphy y Thomaz, S. M. (2008). Global diversity of aquatic macrophytes in freshwater. Hydrobiologia 595 (1): 9-26.

Cirujano, S. y López Alberca, M. D. (1984). Ecología de Najas marina L. en la Mancha. Anales del Fardín Botánico de Madrid 40 (2): 415-419.

Cook, C. D. K. y Urmi-König, K. (1984). A revision of the genus Egeria (Hydrocharitaceae). Aquatic Botany 19: 73-96.

Cook, C. D. K. y Urmi-König, K. (1985). A revision of the genus Elodea (Hydrocharitaceae). Aquatic Botany 21: 111-156.

Dawson, G. (1960). Sinopsis de las especies argentinas del género Utricularia (Lentibulariaceae). Boletín de la Sociedad Argentina Botánica 8 (3-4): 139-159.

Dawson, G. (1965a). Callitrichaceae. En: A. L. Cabrera (Dir.), Flora de la provincia de Buenos Aires - Parte IV (pp. 125-129). Buenos Aires: Instituto Nacional de Tecnología Agropecuaria.

Dawson, G. (1965b). Lentibulariaceae. En: A. L. Cabrera (Dir.), Flora de la provincia de Buenos Aires - Parte V (pp. 308-312). Buenos Aires: Instituto Nacional de Tecnología Agropecuaria.

de la Sota, E. R. y Jankowski, L. (1996). Isoetaceae. En: H. B. Lahitte, J. A. Hurrell (Eds.), Plantas Hidrófilas de la Isla Martín García (Buenos Aires, República Argentina) (p. 23). La Plata: Comisión de Investigaciones Científicas. 
Diante, V. (1996). Ranunculaceae. En: H. B. Lahitte, J. A. Hurrell (Eds.), Plantas Hidrófilas de la Isla Martín García (Buenos Aires, República Argentina) (pp. 133135). La Plata: Comisión de Investigaciones Científicas.

Eskuche, U. G. (2004). La vegetación de la vega del río Paraná medio superior, Argentina. Folia Botanica et Geobotanica Correntesiana 17: 3-59.

Fabris, H. A. (1965). Elatinaceae. En: A. L. Cabrera (Dir.), Flora de la provincia de Buenos Aires - Parte VI (pp. 228-229). Buenos Aires: Instituto Nacional de Tecnología Agropecuaria.

Fabris, H. A. (1966). Haloragaceae. En: A. L. Cabrera (Dir.), Flora de la provincia de Buenos Aires - Parte I (pp. 332-335). Buenos Aires: Instituto Nacional de Tecnología Agropecuaria.

Facelli Fernández, F., Schneider, B., Zilli, F. (2019). Factors driving seed dispersal in a Neotropical river-floodplain system. Acta Botanica Brasilica 33 (3): 1-9.

Fassett, N. C. (1951). Callitriche in the New World. Rhodora 53: 137-155; 161-182; 185-194; 209-222.

Ferreira, A. F., Mormul, R. P., Thomaz, S. M., Pott, A. y Pott, V. J. (2011). Macrophytes in the Upper Paraná river floodplain: checklist and comparison with other large South American wetlands. Revista de Biología Tropical 59 (2): 541556.

Fontana, J. L. (1991). Las comunidades vegetales de una laguna chaqueña del valle del Río Paraná. Folia Botanica et Geobotanica Correntesiana 6: 1-17.

Franceschi, E. A. (2005). Regeneración de la vegetación herbácea luego de una inundación catastrófica en la llanura de inundación del Río Paraná. (Tesis Doctoral), Universidad Nacional de Rosario, Argentina.

Franceschi, E. A. y Lewis J. P. (1979). Notas sobre la vegetación del valle santafesino del río Paraná (República Argentina). Ecosur 6 (11): 55-82

Franceschi, E. A., Prado, D. E. y Lewis J. P. (1985). Comunidades vegetales y mapa de vegetación. Reserva "El Rico" e islas aledañas. Prov. de Santa Fe, Argentina. Rosario: Editora de la Universidad Nacional de Rosario.

Fritschy, B. A. (2003). Geosistema valle actual del río Paraná en un sector de su curso Medio. (Tesis Doctoral), Universidad del Salvador, Argentina.

Gayol, M. P., Morandeira, N. S. y Kandus, P. (2019). Dynamics of shallow lake cover types in relation to Paraná River flood pulses: assessment with multitemporal Landsat data. Hydrobiologia 833 (1): 9-24.

Grisebach, A. (1879). Symbolae ad Floram Argentinam. Zweite Bearbeitung argentinischer Pfalnzen. Abhandlungen der Königlichen Gesellschaft der Wissenschaften in Göttingen 24: 3-345.

Govaerts, R. (2020). World Checklist of Hydrocharitaceae. Facilitated by the Royal Botanic Gardens, Kew. Recuperado de https://wcsp.science.kew.org

Guaglianone, E. R. (1996). Cyperaceae. En: H. B. Lahitte, J. A. Hurrell (Eds.), Plantas Hidrófilas de la Isla Martín García (Buenos Aires, República Argentina) (pp. 70-96). La Plata: Comisión de Investigaciones Científicas.

Haigh, A. (2009). Neotropical Hydrocharitaceae. In: Milliken, W., Klitgård, B. y Baracat, A. (2009 onwards). Neotropikey - Interactive key and information re- 
sources for flowering plants of the Neotropics. Recuperado de http://www.kew. $\mathrm{org} / \mathrm{science} /$ tropamerica/neotropikey/families/Hydrocharitaceae.htm

Handley, R. J. y Davy, A. J. (2000). Discovery of male plants of Najas marina L. (Hydrocharitaceae) in Britain. Watsonia 23: 331-334.

Handley, R. J. y Davy, A. J. (2002). Seedling root establishment may limit Najas marina L. to sediments of low cohesive strength. Aquatic Botany 73 (2): 129-136.

Haynes, R. R. y Holm-Nielsen, L. B. (2003). Flora Neotropica Monograph 85 - Potamogetonaceae. New York, USA: The New York Botanical Garden Press.

Haynes, R. R., Holm-Nielsen, L. B. y Les D. H. (1998). Najadaceae. En: K. Kubitzki (Ed.), The families and genera of vascular plants - Vol. IV (pp. 301-306). Berlin: Springer-Verlag.

Huang, S-Q., Guo, Y-H., Robert, G. W., Shi, Y-H. y Sun, K. (2001). Mechanism of underwater pollination in Najas marina (Najadaceae). Aquatic Botany 70 (1): 67-78.

Hunziker, A. T. (1966). Adiciones al conocimiento de la Flora del Centro de Argentina. I. Najas marina. Kurtziana 3: 221-231.

Hunziker, A. T. (1970). Sobre una nueva hidrófita Argentina: Elatine Lorentziana Nov-sp. Lorentzia 1: 5-10.

Ito, Y., Tanaka, N., Gale, S. W., Yano, O. y Li, J. (2017). Phylogeny of Najas (Hydrocharitaceae) revisited: Implications for systematics and evolution. Taxon 66 (2): 309-323.

Kalesnik, F. A. (2000). Relación entre las comunidades vegetales de los neoecosistemas de albardón y la heterogeneidad ambiental del Bajo Delta del Río Paraná: Tendencias sucesionales y proyecciones sobre la composición futura. (Tesis Doctoral), Universidad de Buenos Aires, Argentina.

Kandus, P. (1997). Análisis de patrones de vegetación a escala regional en el Bajo Delta Bonaerense del Río Paraná (Argentina). (Tesis Doctoral), Universidad de Buenos Aires, Argentina.

Kandus, P., Kalesnik, F., Borgo, L. y Malvárez, A. I. (2002). La reserva natural "Isla Botija" en el delta del rio Paraná: análisis de las comunidades de plantas y condicionantes ambientales. Parodiana 12 (1-2), 3-20.

Kiverling. M. A. (2001). Flora Fanerogámica de la Reserva Ecológica de la Ciudad Universitaria "Paraje El Pozo" Santa Fe, Argentina. (Tesis de Licenciatura), Universidad Nacional del Litoral, Argentina.

Lallana, V. H., Marta, M. C. y Sabattini, R. A. (1981). Macrófitas Acuáticas del Valle Aluvial del Río Paraná Medio. Revisión crítica. En: [s.d. editor], Estudios ecológicos del río Paraná Medio - Vol. II, $1^{\circ}$ parte (pp. 81-135). Santo Tomé: Instituto Nacional de Limnología.

Latrubesse, E. M. (2008). Patterns of anabranching channels: The ultimate endmember adjustment of mega rivers. Geomorphology 101 (1-2): 130-145.

Les, D. H., López Peredo, E., King, U. M., Benoit, L. K., Tippery, N. P., Ball, C. J. y Shannon, R. K. (2015). Through thick and thin: Cryptic sympatric speciation in the submersed genus Najas (Hydrocharitaceae). Molecular phylogenetics and evolution 82: 15-30. 
López Peredo, E., Les, D.H., Benoit, L. y Tippery, N. (2011). Cryptic speciation in Najas marina. En: Stogran J. (Dir.), Botany 2011. Healing the planet (p. 181). Saint Louis: SFB - AFS - ASPT - BSA.

Lourteig, A. (1951). Ranunculáceas de Sudamérica templada. Darwiniana 9 (3/4): 397-608.

Lowden, R. M. (1978). Studies on the submerged genus Ceratophyllum L. in the Neotropics. Aquatic Botany 4: 127-142.

Lowden, R. M. (1986). Taxonomy of the genus Najas L. (Najadaceae) in the neotropics. Aquatic Botany 24 (2): 147-184.

Macluf, C. (2016). Isoëtaceae. En: M. M. Ponce, M. D. Arana (Coords.), Flora Argentina - Vol. 2 (pp. 3-9). San Isidro: Instituto de Botánica Darwinion.

Madanes, N., Quintana, R. D., Kandus, P. y Bó, R. F. (2015). Species richness and functional groups of angiosperms from the Paraná River Delta region (Argentina). Check List 11 (6): 1-12.

Malvárez, A. I. (1997). Las comunidades vegetales del Delta del Río Paraná. Su relación con factores ambientales y patrones del paisaje. (Tesis Doctoral), Universidad de Buenos Aires, Argentina.

Marchetti, Z. Y. (2004). Estudio fitosociológico de la Isla Chapetón (Dpto. Paraná, Entre Ríos). (Tesis de Licenciatura), Universidad Nacional del Litoral, Argentina.

Marchetti, Z. Y. (2011). Patrones de distribución de la vegetación en un tramo de la planicie de inundación del Bajo Paraná. (Tesis Doctoral), Universidad Nacional de Cuyo, Argentina.

Marchetti, Z. Y., Ramonell, C. G., Brumnich, F., Alberdi, R. y Kandus, P. (2020). Vegetation and hydrogeomorphic features of a large lowland river: NDVI patterns summarizing fluvial dynamics and supporting interpretations of ecological patterns. Earth Surface Processes and Landforms 45 (3): 694-706.

Marozzi de Lazzarini, M. C. y de la Peña, M. R. (1981). Documentación fotográfica y fímica de Unidades de Ambiente Flora y Fauna del Paraná Medio (pp. 1-121). Santa Fe: Agua y Energía Eléctrica - Sociedad del Estado.

Marta, M. C. (1977). Nota sobre las hidrófitas de la laguna «Los Matadores»(S. Fe, Argentina). Revista de la Asociación de Ciencias Naturales del Litoral 1 (8): 77-83.

Marta, M. C. (1983). Plantas Acuáticas del Litoral. Santo Tomé, Argentina: Asociación de Ciencias Naturales del Litoral.

Mayora, G., Scarabotti, P., Schneider, B., Alvarenga, P. y Marchese, M. (2020). Multiscale environmental heterogeneity in a large river-floodplain system. Fournal of South American Earth Sciences 100: 102546. doi: https://doi.org/10.1016/ j.jsames.2020.102546.

Mereles, F. y Degen, R. (1993). Haloragaceae. En: R. Spichiger, L. Ramella (Dirs.), Flora del Paraguay 19 (pp. 7-14). Genève: Héliographia.

Minotti P., Ramonell, C. G. y Kandus, P. (2013). Regionalización del corredor fluvial Paraná-Paraguay. En: L. Benzaquén, D. E. Blanco, R. F. Bó, P. Kandus, G. F. Lingua, P. Minotti, R. D. Quintana, S. Sverlij y L. Vidal (Eds.), Inventario de los humedales de Argentina. Sistemas de paisajes de humedales del Corredor Fluvial 
Paraná-Paraguay (pp. 35-90). Buenos Aires: Secretaría de Ambiente y Desarrollo Sustentable de la Nación.

Molero, J. (1985). Ranunculaceae. En: R. Spichiger, G. Bocquet (Dirs.), Flora del Paraguay 3 (pp. 7-25). Genève: Conservatoire et Jardin Botaniques de la Ville de Genève.

Morandeira, N. S. (2014). Tipos funcionales de plantas en humedales de la planicie de inundación del Bajo Río Paraná (Entre Ríos, Argentina) y su observación con datos polarimétricos de radar. (Tesis Doctoral), Universidad de Buenos Aires, Argentina.

Morello, J. (1949). Las comunidades vegetales de las islas cercanas al Puerto de Rosario. (Tesis Doctoral), Universidad Nacional de La Plata, Argentina.

Múlgura, M. E. (1996). Najadaceae. En: F. O. Zuloaga y O. Morrone (Eds.), Catálogo de las Plantas Vasculares de la República Argentina I (p. 242). St. Louis: Missouri Botanical Garden Press.

Negritto, M. A. y Anton, A. M. (1996). Haloragaceae. En: A. T. Hunziker (Dir.), Flora Fanerogámica Argentina 27 (pp. 1-4). Buenos Aires: ProFlora CONICET.

Neiff, J. J. (1978). Fluctuaciones de la vegetación acuática en ambientes del valle de inundación del Paraná medio. Physis 38 (95): 41-53.

Neiff, J. J. (1986). Aquatic plants of the Paraná system. En: B. R. Davies, K. F. Walker (Eds.), The ecology of River Systems (pp. 557-571). Dordrecht: Dr. W. Junk Publishers.

Neiff, J. J. y Malvárez, A. I. (2004). Grandes Humedales Fluviales. En: A. I. Malvárez y R. F. Bó (Comps.), Documentos del Curso Taller Bases Ecológicas para la clasificación e inventario de humedales en Argentina (pp. 77-85). Buenos Aires: Talleres Gráficos Leograf.

Neiff J. J., Casco S. L., Mari E. K. A., Di Rienzo J. A. y Poi, A. S. (2014). Do aquatic plant assemblages in the Paraná River change along the river's length? Aquatic Botany 114: 50-57.

Nicora, E. G. (1968). Najas marina en la mesopotamia argentina. Darwiniana 14: 661.

Oakley L., Prado, D. E. y Adámoli, J. (2005). Aspectos Biogeográficos del Corredor Fluvial Paraguay-Paraná. Miscelánea INSUGEO 14: 245-258.

Orchard, A. E. (1981). A revision of South American Myriophyllum (Haloragaceae) and its repercussions on some Australian and North American species. Brunonia 4 (1): 27-65.

Ørgaard, M. (1991). The genus Cabomba (Cabombaceae) - a taxonomic study. Nordic Fournal of Botany 11 (2): 179-203.

Ospina, J. C. y Zanotti, C. A. (2015a). Cabombaceae. En: F. O. Zuloaga, M. J. Belgrano y A. M. Anton (Eds.), Flora Argentina - Vol. 15 (pp. 31-32). Buenos Aires: Estudio Sigma.

Ospina, J. C. y Zanotti, C. A. (2015b). Ceratophyllaceae. En: F. O. Zuloaga, M. J. Belgrano y A. M. Anton (Eds.), Flora Argentina - Vol. 15 (pp. 33-35). Buenos Aires: Estudio Sigma.

Pastore, A. L. (1936). Las Isoëtaceas argentinas. Revista del Museo de La Plata (nueva serie) 1 (1): 3-30. 
Pedersen, T. M. (1968). Cyperaceae. En: A. L. Cabrera (Dir.), Flora de la provincia de Buenos Aires - Parte I (pp. 315-421). Buenos Aires: Instituto Nacional de Tecnología Agropecuaria.

Pedersen, T. M. (2005). Apiaceae. En: N. M. Bacigalupo (Dir.) Flora Ilustrada de Entre Ríos, Vol. VI, Parte IV B (pp. 544-598). Buenos Aires: Instituto Nacional de Tecnología Agropecuaria.

Peltzer, P. M. (1998). Inventario de flora y fauna del área natural protegida islote municipal del valle aluvial del Paraná. Natura Neotropicalis 1 (29): 74-78.

Pérez del Viso, R., Tur, N. M. y Mantovani, V. (1968). Estimación de la biomasa de hidrófitos en cuencas isleñas del Paraná Medio. Physis 28 (76): 219-226.

Pérez-Moreau, R. A. (1937). Las umbelíferas argentina del género Lilaeopsis. Lilloa 1: 283-306.

Pontiroli, A. (1965). Umbelliferae. En: A. L. Cabrera (Dir.), Flora de la provincia de Buenos Aires - parte IV (pp. 337-403). Buenos Aires: Instituto Nacional de Tecnología Agropecuaria.

Pontiroli, A. (1967a). Nymphaeaceae. En: A. L. Cabrera (Dir.), Flora de la provincia de Buenos Aires - Parte III (pp. 237-241). Buenos Aires: Instituto Nacional de Tecnología Agropecuaria.

Pontiroli, A. (1967b). Ceratophyllaceae. En: A. L. Cabrera (Dir.), Flora de la provincia de Buenos Aires - Parte III (pp. 241-243). Buenos Aires: Instituto Nacional de Tecnología Agropecuaria.

Pontiroli, A. (1967c). Ranunculaceae. En: A. L. Cabrera (Dir.), Flora de la provincia de Buenos Aires - Parte III (pp. 243-257). Buenos Aires: Instituto Nacional de Tecnología Agropecuaria.

Pott, V. J. y Pott, A. (2000). Plantas aquáticas do Pantanal. Brasilia, Brasil: EMBRAPA Comunicação para transferência de Tecnologia.

Ramonell, C. G., Kandus, P., Minotti, P., Borro, M. y Morandeira, N. S. (2012). Nueva interpretación geomorfológica del complejo fluviolitoral del río Paraná en su desembocadura. En: S. Degiovanni, M. J. Andreazzini (Coords.), Actas de resúmenes del V Congreso Argentino de Cuaternario y Geomorfología (pp. 125-126). Río Cuarto: UniRío Editora.

Rodríguez, E. E. (2007). Catálogo florístico del Parque Nacional Pre-Delta (Entre Ríos, Argentina). (Tesis de Licenciatura), Universidad Autónoma de Entre Ríos, Argentina.

Rodríguez, E. E., Senkman, E. y Aceñolaza, P. G. (2006). Catálogo Florístico de una Reserva Ecológica Urbana: Islote Municipal, Paraná (Entre Ríos, Argentina). Natura Neotropicalis 1 (37): 67-75.

Sabattini, R. y Lallana V. H. (2007). Aquatic macrophytes. En: Iriondo M. H., Paggi J. C., Parma M. J. (Eds.). The Middle Paraná River - Limnology of a Subtropical Wetland (pp. 205-226). Berlin: Springer-Verlag.

Sabattini, R. A., Lallana, V. H., y Marta, M. C. (1983). Inventario y biomasa de plantas acuáticas en un tramo del valle aluvial del río Paraná Medio. Revista de la Asociación de Ciencias Naturales del Litoral 2 (14): 179-191. 
Schneider, B. (2017). Ensambles de macrófitas en ambientes de la llanura aluvial del río Paraná Medio: Factores que inciden a distintas escalas. (Tesis Doctoral), Universidad Nacional del Litoral, Argentina.

Senkman, E. (2005). Catálogo de plantas del herbario de la isla Puente (pp. 1-4). Paraná: Museo de Ciencias Naturales y Antropológicas "Prof. Antonio Serrano".

Sirolli, L. H. (2018). Regeneración de especies nativas en los bosques de albardón del Delta Frontal del río Paraná (Buenos Aires, Argentina). (Tesis Doctoral), Universidad de Buenos Aires, Argentina.

St. John, H. (1961). Monograph of the genus Egeria Planchon. Darwiniana 12: 293307.

St. John, H. (1963). Monograph of the genus Elodea (Hydrocharitaceae). 3. The species in Northern and Eastern South America. Darwiniana 12: 639-652.

Stanković, Ž., Borišev, M., Simić S., Vuèković, M., Igić, R., Vidović, M. y Miljanović B. (2009). Macrophytes of the Grlište reservoir (Serbia): fifteen years after its establishment. Archives of Biological Sciences 61 (2): 267-278. doi:10.2298/ ABS0901267S

Talavera, S. y Gallego, M. J. (2010). Najas L. En: S. Talavera, M.J. Gallego, C. Romero Zarco, A. Herrero (Eds.), Flora Iberica XVII. Butomaceae-funcaceae (pp. 55-62). Madrid: Consejo Superior de Investigaciones Científicas.

Thiers, B. (2020). Index Herbariorum: A global directory of public herbaria and associated staff. New York Botanical Garden's Virtual Herbarium. Recuperado de http://sweetgum.nybg.org/ih/

Triest, L. (1987). A revision of the genus Najas L. (Najadaceae) in Africa and surrounding islands. Mémoires de l'Académie Royale des Sciences d'Outre-Mer. Classe des Sciences Naturelles et Médicales (NS) 21 (4): 1-89.

Triest, L. (1988). A revision of the genus Najas L. (Najadaceae) in the Old World. Mémoires de l'Académie Royale des Sciences d'Outre-Mer. Classe des Sciences Naturelles et Médicales (NS) 22 (1): 1-172.

Torres, M. A. (1968). Zannichelliaceae. En: Cabrera, A. L. (Dir.), Flora de la provincia de Buenos Aires - Parte I (pp. 288-290). Buenos Aires: Instituto Nacional de Tecnología Agropecuaria.

Tur, N. M. (1968). Potamogetonaceae. En: Cabrera, A. L. (Dir.), Flora de la provincia de Buenos Aires - Parte I (pp. 279-288). Buenos Aires: INTA.

Tur, N. M. (1970). Notas sobre Hidrofitos e Higrófilos Argentinos. Revista de la Asociación de Ciencias Naturales del Litoral 1 (1): 13-14.

Tur, N. M. (1972). Embalsados y Camalotes de la región isleña del Paraná Medio. Darwiniana 17: 397-407.

Tur, N. M. (1982). Revisión del género Potamogeton L. en la Argentina. Darwiniana 24 (1-4): 217-265.

Tur, N. M. (1987b). Cabombaceae. En: N. Troncoso y N. M. Bacigalupo (Dirs.), Flora Ilustrada de Entre Ríos - Parte III (pp. 303-304). Buenos Aires: Instituto Nacional de Tecnología Agropecuaria.

Tur, N. M. (1987a). Ceratophyllaceae. En: N. Troncoso y N. M. Bacigalupo (Dirs.), Flora Ilustrada de Entre Ríos - Parte III (pp. 300-303). Buenos Aires: Instituto Nacional de Tecnología Agropecuaria. 
Tur, N. M. (1990). Potamogetonaceae. En: R. Spichiger, L. Ramella (Dirs.), Flora del Paraguay 15 (pp. 7-14). Genève: Héliographia.

Tur, N. M. (1995). Ceratophyllaceae. En: A. T. Hunziker (Dir.), Flora Fanerogámica Argentina 6 (pp. 1-2). Buenos Aires: ProFlora CONICET.

Tur, N. M. (1996a). Potamogetonaceae. En: H. B. Lahitte, J. A. Hurrell (Eds.), Plantas Hidrófilas de la Isla Martín García (Buenos Aires, República Argentina) (p. 34). La Plata: Comisión de Investigaciones Científicas.

Tur, N. M. (1996b). Hydrocharitaceae. En: H. B. Lahitte, J. A. Hurrell (Eds.), Plantas Hidrófilas de la Isla Martín García (Buenos Aires, República Argentina) (pp. 41-42). La Plata: Comisión de Investigaciones Científicas.

Tur, N. M. (1996c). Haloragaceae. En: H. B. Lahitte, J. A. Hurrell (Eds.), Plantas Hidrófilas de la Isla Martín García (Buenos Aires, República Argentina) (p. 169). La Plata: Comisión de Investigaciones Científicas.

Tur, N. M. (1996d). Umbelliferae. En: H. B. Lahitte, J. A. Hurrell (Eds.), Plantas Hidrófilas de la Isla Martín García (Buenos Aires, República Argentina) (pp. 170175). La Plata: Comisión de Investigaciones Científicas.

Tur, N. M. (2005). Haloragaceae. En: N. M. Bacigalupo (Dir.), Flora Ilustrada de Entre Ríos - Parte IV B (pp. 541-543), Lugar, Editorial.

Tur, N. M. (2008a). Hydrocharitaceae. En: J. A. Hurrell (Dir.), Flora Rioplatense - Parte III Vol. 1 (pp. 177-198). Buenos Aires: Editorial Literature Of Latin America.

Tur, N. M. (2008b). Potamogetonaceae. En: J. A. Hurrell (Dir.), Flora Rioplatense - Parte III Vol. 1 (pp. 289-300). Buenos Aires: Editorial Literature Of Latin America.

Tur, N. M. (2008c). Zannichelliaceae. En: J. A. Hurrell (Dir.), Flora Rioplatense - Parte III Vol. 1 (pp. 301-306). Buenos Aires: Editorial Literature Of Latin America.

Tur, N. M. y Lavigne, A. (1992). Plantas acuáticas de la provincia de Buenos Aires. En: H. L. López, E. P. Tonni (Coords.), Situación Ambiental de la Provincia de Buenos Aires (pp. 1-30). La Plata: Comisión de Investigaciones Científicas.

Urquiola Cruz, A. J., Vega Hernández, E., Novo Carbó, R. y López M. L. (1998). Las Najadaceae de Cuba. Anales del Fardín Botánico de Madrid 56 (1): 85-94.

Villalba, A. B. (2015). Cambios de la vegetación en el delta del Arroyo Leyes. (Tesis de Licenciatura), Universidad Nacional del Litoral, Argentina.

Wang, Q. F., Youhao, G., Haynes, R. R. y Hellquist, C. B. (2010). Hydrocharitaceae. En: Z. Wu, P. H. Raven (Eds.), Flora of China 23: Acoraceae through Cyperaceae (pp. 91-102). Beijing y St. Louis: Science Press y Missouri Botanical Garden Press.

Zhang, L. (Ed.). (2010). Flora of China Illustrations 23: Acoraceae through Cyperaceae. Beijing, China y St. Louis, USA: Science Press y Missouri Botanical Garden Press.

Zuloaga, F. O., Belgrano, M. J. y Zanotti, C. A. (2019). Actualización del Catálogo de las Plantas Vasculares del Cono Sur. Darwiniana nueva serie 7 (2): 208-278. Base de datos recuperada de http://conosur.floraargentina.edu.ar/ 
Apéndice 1. Bibliografía utilizada para compilar las especies de hidrófitas sumergidas reportadas en la Tabla 1. En la columna "Tipo de fuente" (ver materiales y métodos) las referencias son: LFO = lista florística original para la planicie de inundación del Bajo Paraná; RF = Revisión de listas florísticas; $\mathrm{TT}=$ tratamientos taxonómicos que se ordenan en la columna "Taxón"; nc = no corresponde.

Appendix 1. Bibliography used to compile the submersed hydrophytes species reported in Table 1. In the column "Source type" (see Materials and methods) the references are: LFO = original floristic list for the Lower Paraná floodplain; RF = floristic list revision; TT = taxonomic treatments which are ordered in the "Taxon" column; nc = not applicable.

\begin{tabular}{|c|c|c|}
\hline Cita & Tipo de fuente & Taxón \\
\hline Aceñolaza et al. (2008) & RF & n.c. \\
\hline Affolter (1985) & TT & Lilaeopsis \\
\hline Arbo (2002) & TT & Utricularia \\
\hline Bacigalupo (2005) & TT & Elatine \\
\hline Bacigalupo (1979a) & TT & Callitriche \\
\hline Bacigalupo (1979b) & TT & Callitriche \\
\hline Bacigalupo (1987) & TT & Ranunculus \\
\hline Bacigalupo (1970) & TT & Elatine \\
\hline Barros (1947) & TT & Eleocharis \\
\hline Bayo et al. (1981) & LFO & n.c. \\
\hline Botta (1979) & TT & Utricularia \\
\hline Brumnich (2017) & LFO & n.c. \\
\hline Burkart (1957) & LFO & n.c. \\
\hline Cabrera (1968) & TT & Egeria / Elodea \\
\hline Capurro (1968) & TT & Isoetes \\
\hline Casco $(2003)$ & LFO & n.c. \\
\hline Cavallaro y Tobío (2017) & LFO & n.c. \\
\hline Cook y Urmi-König (1984) & TT & Egeria / Elodea \\
\hline Cook y Urmi-König (1985) & TT & Egeria / Elodea \\
\hline Dawson (1965a) & TT & Callitriche \\
\hline Dawson (1965b) & TT & Utricularia \\
\hline Dawson (1960) & TT & Utricularia \\
\hline de la Sota y Jankowski (1996) & TT & Isoetes \\
\hline Diante (1996) & TT & Ranunculus \\
\hline Eskuche (2004) & LFO & n.c. \\
\hline Fabris (1966) & TT & Myriophyllum \\
\hline Fabris (1965) & TT & Elatine \\
\hline Facelli Fernández et al. (2019) & LFO & n.c. \\
\hline Fassett (1951) & TT & Callitriche \\
\hline Fontana (1991) & LFO & n.c. \\
\hline Franceschi y Lewis (1979) & LFO & n.c. \\
\hline Franceschi et al. (1985) & LFO & n.c. \\
\hline Franceschi (2005) & LFO & n.c. \\
\hline Fritschy (2003) & LFO & n.c. \\
\hline Gayol et al. (2019) & LFO & n.c. \\
\hline Guaglianone (1996) & TT & Eleocharis \\
\hline Haynes y Holm-Nielsen (2003) & TT & Potamogetonaceae \\
\hline Hunziker (1970) & TT & Elatine \\
\hline Kalesnik (2000) & LFO & n.c. \\
\hline Kandus (1997) & LFO & n.c. \\
\hline Kandus et al. (2002) & LFO & n.c. \\
\hline Kiverling (2001) & LFO & n.c. \\
\hline Lallana et al. (1981) & $\mathrm{RF}$ & n.c. \\
\hline Lourteig (1951) & TT & Ranunculus \\
\hline Lowden (1978) & TT & Ceratophyllum \\
\hline Macluf (2016) & TT & Isoetes \\
\hline Madanes et al. (2015) & $\mathrm{RF}$ & n.c. \\
\hline Malvárez (1997) & LFO & n.c. \\
\hline
\end{tabular}


Apéndice 1 (cont.)

\begin{tabular}{|c|c|c|}
\hline Cita & Tipo de fuente & Taxón \\
\hline Marchetti (2004) & LFO & n.c. \\
\hline Marchetti (2011) & LFO & n.c. \\
\hline Marozzi de Lazzarini y de la Peña (1981) & LFO & n.c. \\
\hline Marta (1977) & LFO & n.c. \\
\hline Marta (1983) & $\mathrm{RF}$ & n.c. \\
\hline Mereles y Degen (1993) & TT & Myriophyllum \\
\hline Molero (1985) & TT & Ranunculus \\
\hline Morandeira (2014) & LFO & n.c. \\
\hline Morello (1949) & LFO & n.c. \\
\hline Negritto y Antón (1996) & TT & Myriophyllum \\
\hline Neiff (1978) & LFO & n.c. \\
\hline Neiff (1986) & $\mathrm{RF}$ & n.c. \\
\hline Neiff et al. (2014) & $\mathrm{RF}$ & n.c. \\
\hline Orchard (1981) & TT & Myriophyllum \\
\hline Ørgaard (1991) & TT & Cabomba \\
\hline Ospina y Zanotti (2015a) & TT & Cabomba \\
\hline Ospina y Zanotti (2015b) & TT & Ceratophyllum \\
\hline Pastore (1936) & TT & Isoetes \\
\hline Pedersen (1968) & TT & Eleocharis \\
\hline Pedersen (2005) & TT & Lilaeopsis \\
\hline Peltzer (1998) & LFO & n.c. \\
\hline Pérez del Viso et al. (1968) & LFO & n.c. \\
\hline Pérez-Moreau (1937) & TT & Lilaeopsis \\
\hline Pontiroli (1965) & TT & Lilaeopsis \\
\hline Pontiroli (1967b) & TT & Ceratophyllum \\
\hline Pontiroli (1967a) & TT & Cabomba \\
\hline Pontiroli (1967c) & TT & Ranunculus \\
\hline Rodríguez (2007) & LFO & n.c. \\
\hline Rodríguez et al. (2006) & LFO & n.c. \\
\hline Sabattini et al. (1983) & RF & n.c. \\
\hline Sabattini y Lallana (2007) & $\mathrm{RF}$ & n.c. \\
\hline Schneider (2017) & LFO & n.c. \\
\hline Senkman (2005) & LFO & n.c. \\
\hline Sirolli (2018) & LFO & n.c. \\
\hline St. John (1961) & TT & Egeria / Elodea \\
\hline St. John (1963) & TT & Egeria / Elodea \\
\hline Torres (1968) & TT & Zannichellia \\
\hline Tur (1968) & TT & Potamogetonaceae \\
\hline Tur (1972) & LFO & n.c. \\
\hline Tur (1982) & TT & Potamogetonaceae \\
\hline Tur (1987b) & TT & Cabomba \\
\hline Tur (1987a) & TT & Ceratophyllum \\
\hline Tur (1990) & TT & Potamogetonaceae \\
\hline Tur (1995) & TT & Ceratophyllum \\
\hline Tur (1996a) & TT & Potamogetonaceae \\
\hline Tur (1996b) & TT & Egeria / Elodea \\
\hline Tur (1996c) & TT & Myriophyllum \\
\hline Tur (1996d) & TT & Lilaeopsis \\
\hline Tur (2005) & TT & Myriophyllum \\
\hline Tur (2008a) & TT & Egeria / Elodea \\
\hline Tur (2008b) & TT & Potamogetonaceae \\
\hline Tur $(2008 c)$ & TT & Zannichellia \\
\hline Tur y Lavigne (1992) & $\mathrm{RF}$ & n.c. \\
\hline Villalba (2015) & LFO & n.c. \\
\hline
\end{tabular}

